\title{
A velocity map imaging detector with an integrated gas injection system
}

\author{
O. Ghafur, ${ }^{1}$ W. Siu, ${ }^{1}$ P. Johnsson, ${ }^{1}$ M. F. Kling, ${ }^{1,2}$ M. Drescher, ${ }^{3}$ and M. J. J. Vrakking ${ }^{1}$ \\ ${ }^{1}$ FOM-Institute for Atomic and Molecular Physics (AMOLF), Kruislaan 407, 1098 SJ Amsterdam, \\ The Netherlands \\ ${ }^{2}$ Max-Planck-Institut für Quantenoptik, Hans-Kopfermann-Str. 1, 85748 Garching, Germany \\ ${ }^{3}$ Institut für Experimentalphysik, Universität Hamburg, Luruper Chaussee 149, D-22761 Hamburg, Germany
}

(Received 4 November 2008; accepted 29 January 2009; published online 18 March 2009)

\begin{abstract}
We present the design of a velocity map imaging spectrometer where the target gas is injected from a capillary that is integrated in the repeller plate of the ion optics assembly that drives electrons/ions formed by ionization or dissociation to a two-dimensional detector. The geometry of this design allows the use of gas densities in the interaction region that are two to three orders of magnitude higher than the densities that are used in standard velocity map imaging spectrometers, making the detector suitable for working with weak light sources such as newly developed attosecond pulse sources, or (quasi-)cw sources such as synchrotrons. In a test where monoenergetic photoelectrons were generated by six-photon ionization of Xe (utilizing the second harmonic of a neodymium doped Nd:YAG), the kinetic energy resolution of the spectrometer was found to be $\Delta E / E=1.8 \%$. This number was found to be in good agreement with Monte Carlo simulations. (C) 2009 American Institute of Physics. [DOI: 10.1063/1.3085799]
\end{abstract}

\section{INTRODUCTION}

Since its first introduction by Chandler and Houston ${ }^{1}$ in 1990, two-dimensional (2D) ion/photoelectron imaging has become a popular tool for measuring angle- and energyresolved ion or electron velocity distributions that result from photoionization or molecular photodissociation. ${ }^{2}$ In $2 \mathrm{D}$ imaging experiments, ions or electrons are projected onto a 2D detector that typically consists of a microchannel plate (MCP) detector followed by a phosphor screen and a camera system or-alternatively - a MCP detector followed by a delay line or resistive anode encoder where the position information is electronically registered. In the original design of Chandler and Houston, ${ }^{1}$ the fragmentation and ionization process was performed in a flat field between a repeller and a pair of grids. In a major improvement in the technique, Eppink and Parker ${ }^{3}$ exchanged the grid for an open electrode, leading to a situation where the ion optics design incorporates a lens. Upon proper tuning of this lens, the position where the ions/electrons impinge on the $2 \mathrm{D}$ detector is almost exclusively determined by their velocity and hardly by the departure point of their trajectory. Hence, the term "velocity map imaging" was introduced for this technique. In the years since its development, velocity map imaging has become a very popular experimental technique that is finding application in an ever increasing number of areas, ${ }^{2}$ such as attosecond science ${ }^{4}$ and experiments at emerging (soft-)x-ray free electron lasers. ${ }^{5}$ Using velocity map imaging moderately high-resolution $(\Delta E / E \cong 1 \%)$ kinetic energy spectra and angular distributions can be efficiently collected at high count rates. Depending on the size of the interaction region, $10^{2}-10^{4}$ counts/laser shot can typically be collected without space-charge broadening of the measured kinetic energy distributions. Further improvements in the technique have involved various kinds of slice imaging, where the time-gated detection is combined with increasing the spread in the ion/ electron arrival time on the detector (by means of pulsed extraction ${ }^{6,7}$ and/or extraction in a relatively weak dc electric field $\left.^{7}\right)$. This allows one to restrict the detection to a 2D slice of the original three-dimensional (3D) velocity distribution, thereby avoiding the use of a retrieval algorithm for obtaining the $3 \mathrm{D}$ velocity distribution and increasing the kinetic energy resolution.

In all the above-mentioned implementations of 2D imaging, a gaseous sample is introduced into the imaging spectrometer by means of a (skimmed) atomic/molecular beam. The advantage of this approach is that samples are used where the initial velocity is rather well defined. Furthermore, the rotational and, possibly, vibrational cooling of the gas during the supersonic expansion into the vacuum leads to a reduction in the rotational and vibrational temperature of the sample, which is frequently beneficial. A drawback is the fact that relative large amounts of gas need to be introduced into the vacuum system in order to obtain appreciable gas densities in the interaction region. The use of pulsed valves reduces this problem somewhat but becomes problematic when the repetition rate of the experiment is very high $(\geq 1 \mathrm{kHz})$ or-in the most extreme case-if the experiment operates in continuous mode. Low target gas densities are particularly problematic if the light source is very weak.

In our research, we apply $2 \mathrm{D}$ velocity map imaging in experiments with attosecond laser pulses. ${ }^{4}$ The present state of the art allows for the generation of isolated attosecond pulses with a duration of down to about 100 as. ${ }^{8,9}$ However, these pulses typically contain no more than $10^{6}$ photons per pulse. Combining this number of photons with the typical density in an atomic/molecular beam of $10^{13}$ atoms/molecules per cc, and using a typical absorption cross section of $10^{-17} \mathrm{~cm}^{2}$ and a typical atomic/molecular beam width of $10^{-1} \mathrm{~cm}$, this leads to a probability of photo- 


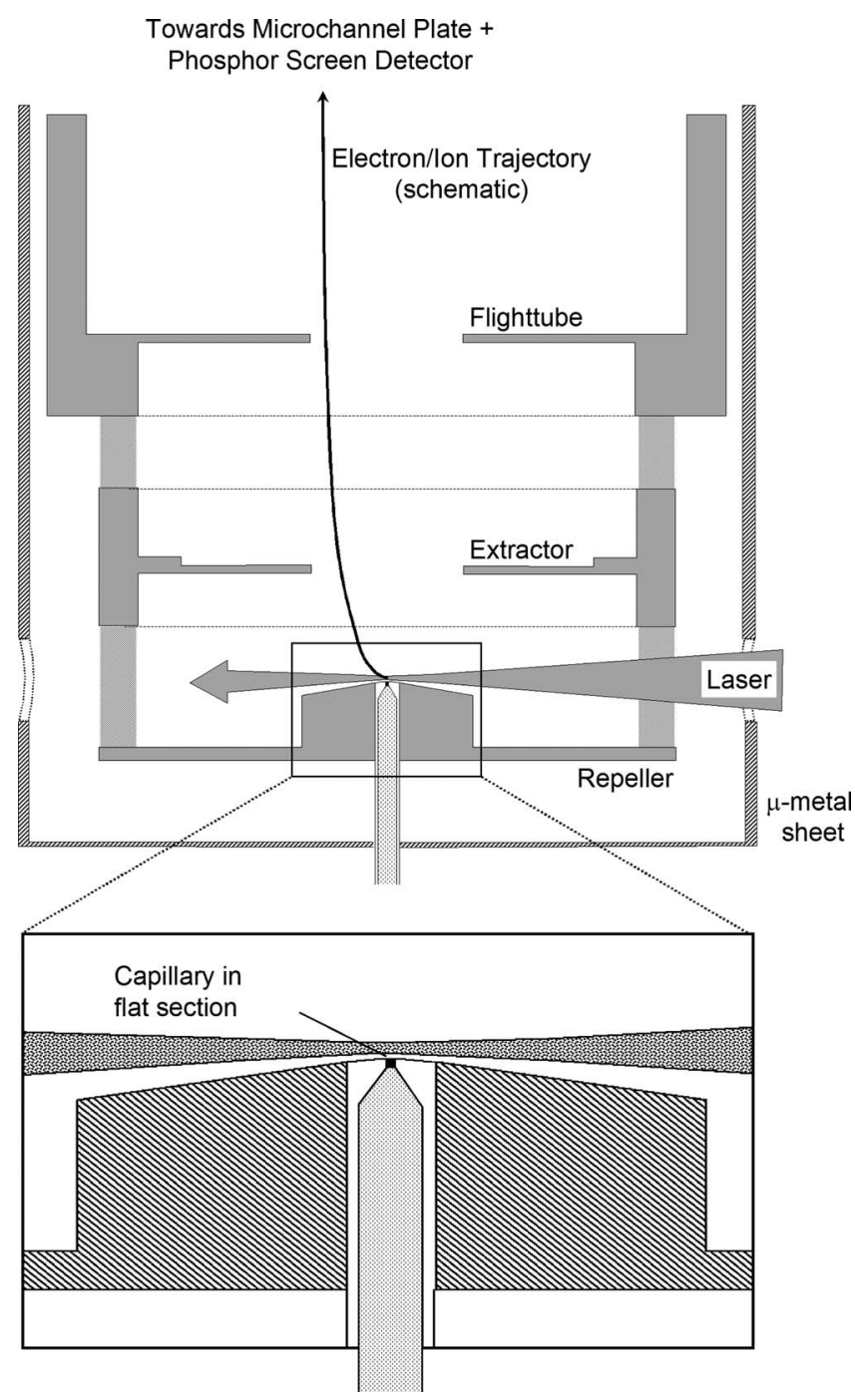

FIG. 1. The velocity map imaging spectrometer introduced in this paper. Ions/electrons are formed at the crossing point of a laser beam and the symmetry axis of the spectrometer and are accelerated toward a MCP + phosphor screen assembly by applying appropriate voltages to repeller and extractor electrodes. In a conventional velocity map imaging spectrometer, the repeller electrode is a flat plate, and the gas typically enters the detector through a small hole drilled in the repeller electrode (i.e., along the symmetry axis of the spectrometer) or from the side (i.e., along an axis that lies in a plane perpendicular to the detector axis, and containing the laser beam). In our new design, the repeller electrode incorporates a $50 \mu \mathrm{m}$ capillary tube from which the gas escapes in close proximity to the laser focus. The central $\phi=1 \mathrm{~mm}$ part of the repeller electrode is flat (i.e., parallel to the detection plane). From there the repeller electrode assumes a conical shape with a $154^{\circ}$ cone angle to accommodate focusing of the laser in front of the capillary.

absorption of $\sim 10^{-5}$ (i.e., typically $<10$ photoabsorptions per laser pulse, which is too low for many applications). Therefore in this paper, in the spirit of earlier work by Samartzis ${ }^{10}$ and Toomes and Kitsopoulos, ${ }^{11}$ we introduce a new spectrometer design where the gas that is to be used in the experiment flows out of a capillary that is integrated in the repeller electrode of the spectrometer and that is produced by laser machining (see Fig. 1). Using this arrangement, the gas density that can be maintained in the interaction region is only limited by the maximum pressure that can be tolerated in the experimental chamber. Typically, this pressure is limited by the fact that a MCP detector needs to be operated at high gain in this chamber. Assuming a $500 \mathrm{l} / \mathrm{s}$ pump and a maximum tolerable pressure of $10^{-5}$ Torr, this suggests that $5 \times 10^{-3}$ Torr $1 / \mathrm{s}$ can be introduced into the vacuum system, corresponding to 1.5 $\times 10^{17}$ atoms/molecules per second. Assuming atoms/ molecules moving at a velocity of $300 \mathrm{~m} / \mathrm{s}$ through a capillary with a diameter of $50 \mu \mathrm{m}$, the flow rate is 6 $\times 10^{-4} \mathrm{l} / \mathrm{s}$ and it follows that at the exit of the capillary a pressure of about 8.5 Torr can be maintained. In order to avoid unwanted photoelectron production by surface photoemission (which is a limiting factor in the attosecond experiments that were mentioned previously), we will assume that the experiment is performed at a distance of $500 \mu \mathrm{m}$ from the exit of the capillary, where the density has dropped by a factor 100. Assuming an opening angle of 1 sr for the gas that effuses out of the capillary, the probability for a laser photon to be absorbed is approximately $0.125 \%$, i.e., more than two orders of magnitude higher than in the case when a skimmed molecular beam is used. Further improvements are possible when a pulsed gas source is used in the repeller, which meanwhile has become the preferred mode of operation of the spectrometer in our laboratory. Additionally, the pumping speed of the vacuum system may be increased, or differential pumping may be introduced in the detector region. The latter is challenging, however, given the open geometry that is required in velocity map imaging. Note that apart from the new repeller assembly, the design of the spectrometer was used in our previous work and is described in Ref. 12 .

In this paper the design of the new velocity map imaging spectrometer is presented, and its performance is compared to that of the commonly used Eppink and Parker ${ }^{3}$ design. Experimental results are presented where the detector has been used to measure photoelectron spectra resulting from multiphoton ionization of Xe atoms by a neodymium doped yttrium aluminum garnet (Nd:YAG) laser. Meanwhile, the spectrometer has also been successfully applied in experiments using a $1 \mathrm{KHz}$ source of isolated attosecond pulses, each containing $<10^{6}$ photons/pulse. ${ }^{13,14}$

\section{DESIGN OF A REPELLER WITH AN INTEGRATED GAS INJECTION SYSTEM}

In the traditional Eppink and Parker $^{3}$ design, the extraction region of the velocity map imaging spectrometer consists of a flat plate that acts as a repeller and a ring electrode that acts as the extractor for the electrons or ions that are produced. Due to the hole in the extractor electrodes, the electrons or ions travel through a region where the equipotential lines are curved. It is this curvature that gives rise to the desired velocity mapping. In the Eppink and Parker ${ }^{3}$ design, the distance from the point where the electrons or ions are produced (henceforth called "the interaction region"), and the repeller/extractor electrodes are typically 5-10 $\mathrm{mm}$. However, this is not strictly necessary. Arguably, any electrode geometry that replicates the curved field lines of the open geometry will lead to identical velocity mapping. This is exploited in our present design where the repeller extends very close to the interaction region. By following the field lines of the Eppink and Parker ${ }^{3}$ geometry for an appropriate 

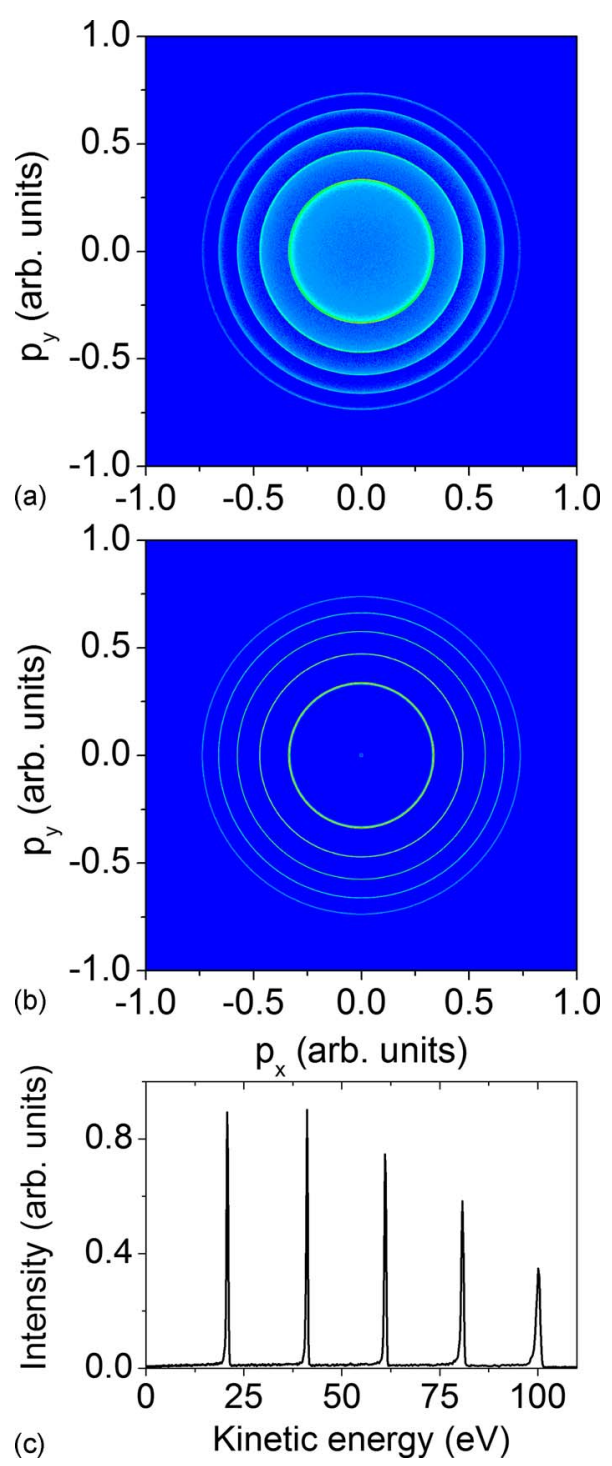

FIG. 2. (Color online) (a) Simulated image determined by performing a Monte Carlo simulation where $20 \times 10^{6}$ particles with kinetic energies of 20 , $40, \ldots, 100 \mathrm{eV}$ were projected onto a 2D detector plane by means of an open electron/ion optics design as introduced by Eppink and Parker (Ref. 3). (b) Slice through the 3D velocity distribution that was obtained from (a) by means of an inverse Abel transform. (c) Kinetic energy distribution derived from (b) allowing an assessment of the energy resolution of the spectrometer.

length (that is, to be determined by means of simulations), it is ensured that electrons or ions produced in the interaction region undergo velocity mapping while at the same allowing for efficient injection of the gas at high density from the repeller electrode. The final design that has been developed is shown in Fig. 1. The repeller electrode consists of a $\phi$ $=1 \mathrm{~mm}$ diameter flat part that ensures that the field lines in the interaction region approximate those of the Eppink and Parker $^{3}$ velocity map imaging spectrometer and then turns into a conical shape in order to allow access to the interaction region with a focused laser beam.

The detailed design of the repeller electrode was performed using a home-built electrostatic trajectory code. No particle collisions are assumed to take place. Making use of the inherent cylindrical symmetry around the electric field axis, the Laplace equation was solved on a $2 \mathrm{D}$ grid $(\delta z, \delta r$

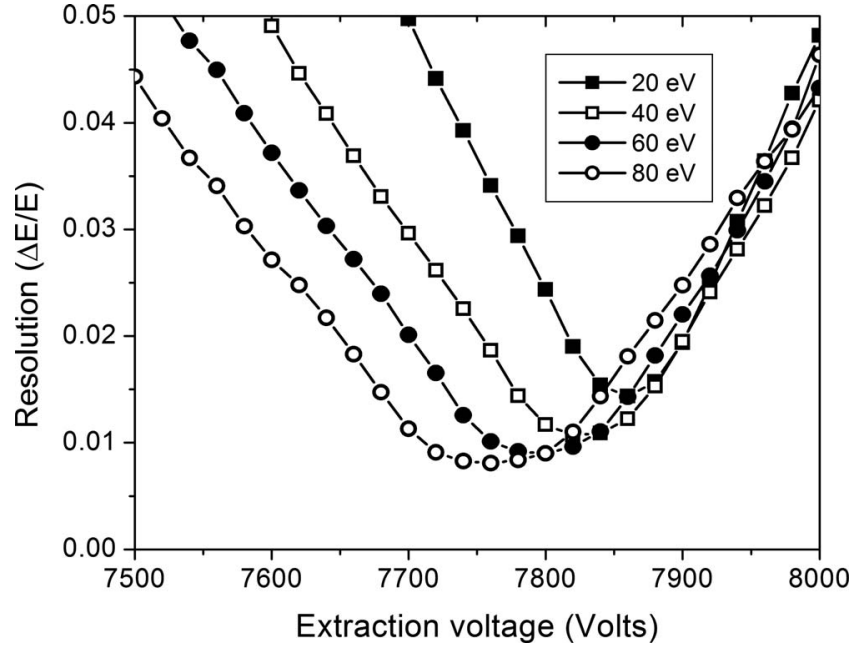

FIG. 3. Calculated energy resolution $\Delta E / E$ for a conventional velocity map imaging spectrometer as function of the kinetic energy of the fragments and the voltage applied to the extractor electrode. The geometry shown in Fig. 1 is assumed, with $10 \mathrm{kV}$ applied to a flat repeller plate and with a $26 \mathrm{~cm}$ separation between the repeller and the detection plane. The ejection of the fragments is assumed to be isotropic. The fragment source volume is assumed to be the intersection of a molecular beam with a Gaussian radial profile ( $2 \mathrm{~mm}$ FWHM) and a Gaussian laser beam that is focused to a spot size (FWHM) of $200 \mu \mathrm{m}$.

$=0.025 \mathrm{~mm})$ using standard numerical techniques. Using Monte Carlo sampling the initial conditions (position and velocity in three dimensions) of $20 \times 10^{6}$ charged particles were chosen, and trajectories leading to impact on a $2 \mathrm{D}$ plane placed $26 \mathrm{~cm}$ downstream from the repeller electrode were evaluated. The initial particle kinetic energies were chosen as $20,40, \ldots, 100 \mathrm{eV}$, and the angular distribution was assumed to be isotropic. The fragment source volume was assumed to be the intersection of a molecular beam with a Gaussian radial profile [2 $\mathrm{mm}$ full width at half maximum (FWHM)] and a Gaussian laser beam that is focused to a spot size (FWHM) of $200 \mu \mathrm{m}$. Calculated 2D distributions, which correspond to an Abel projection of the assumed 3D velocity distribution, were evaluated on a $2000 \times 2000$ grid and were fed into an inversion algorithm ${ }^{15}$ in order to determine the kinetic energy resolution of the instrument at the five kinetic energies chosen. A typical result of this procedure is shown in Fig. 2. In Fig. 2(a), a simulated image is shown from which the 3D velocity distribution [Fig. 2(b)] is derived by means of an inverse Abel transform.

The results shown in Fig. 2 were obtained using the lens design proposed by Eppink and Parker. ${ }^{3}$ This design allows for very high-resolution measurements, as illustrated by Fig. 3, where the calculated kinetic energy resolution $\Delta E / E$ is plotted as a function of the electron kinetic energy and the voltage that is applied to the extractor electrode (while maintaining a constant $10 \mathrm{kV}$ voltage on the repeller electrode). Figure 3 reveals that the lens displays some chromatic aberration, with low kinetic energy particles requiring slightly higher extraction voltages than higher energy particles. Nevertheless, Fig. 3 shows that velocity map imaging allows measurements of fragment kinetic energy spectra with an energy resolution down to $0.7 \%$, in agreement with laboratory experience. We note that the actual value of the reso- 

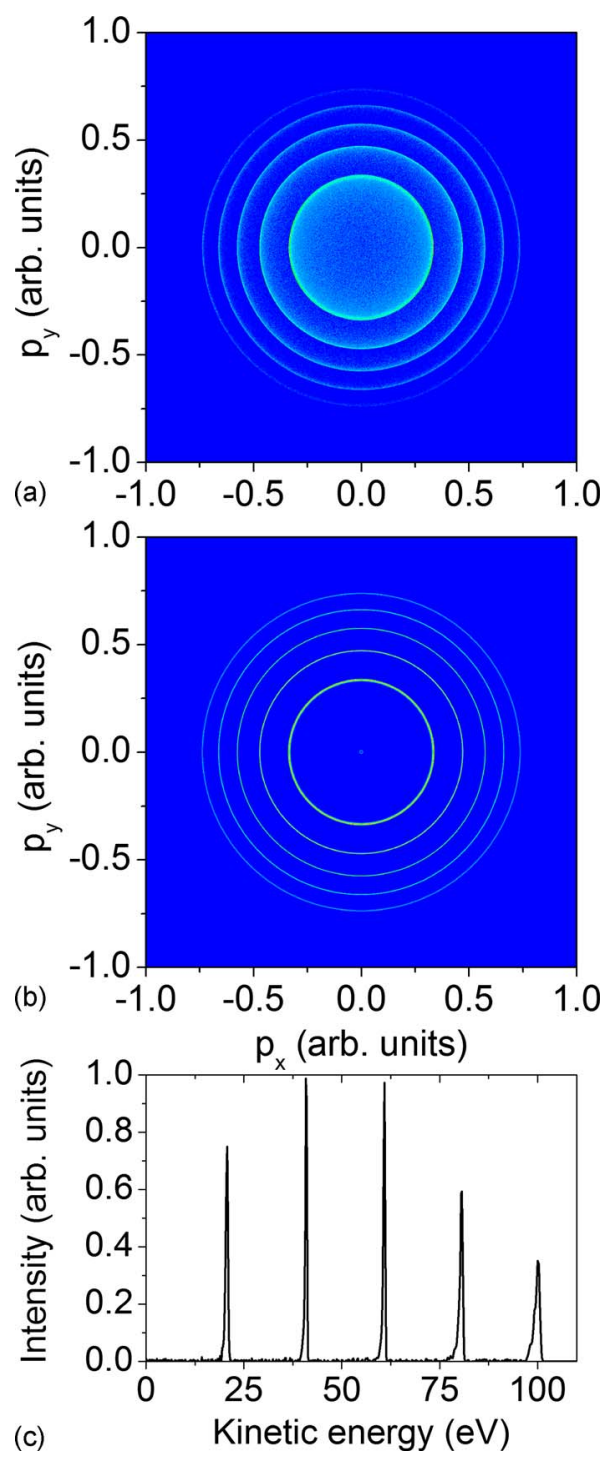

FIG. 4. (Color online) (a) Simulated image determined by performing a Monte Carlo simulation where $20 \times 10^{6}$ particles with kinetic energies of 20 , $40, \ldots, 100 \mathrm{eV}$ were projected onto a $2 \mathrm{D}$ detector plane by means of the new velocity map imaging design that incorporates a capillary in a conical repeller. (b) Slice through the 3D velocity distribution that was obtained from (a) by means of an inverse Abel transform. (c) Kinetic energy distribution derived from (b) allowing an assessment of the energy resolution of the spectrometer.

lution that may be expected in the laboratory is influenced by the choice of the flight length from the interaction region to the $2 \mathrm{D}$ detector, as well as by the size of the interaction volume. Through optimization of these features, Lipciuc et al. ${ }^{16}$ were able to achieve an experimental resolution of $0.38 \%$.

As discussed, the resolution that can be achieved in velocity map imaging is independent of the detailed shape of the electrodes, provided that the fields that are encountered by the particles on their path from the extraction region to the $2 \mathrm{D}$ detector are unchanged, leading us to the design shown in Fig. 1. In contrast with the simple lens design of Eppink and Parker, ${ }^{3}$ we may anticipate that the energy resolution of this new design depends not only on the voltages that are applied to the repeller and extractor electrodes but furthermore on the width of the flat part of the repeller and

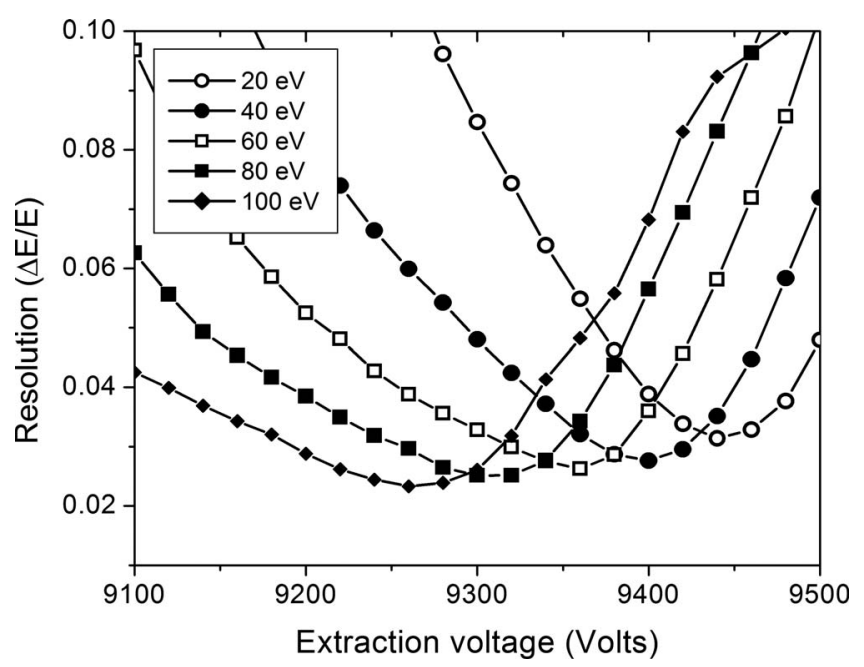

FIG. 5. Calculated energy resolution $\Delta E / E$ for the new velocity map imaging spectrometer where the gas injection is integrated in the repeller electrode as a function of the kinetic energy of the fragments and the voltage applied to the extractor electrode. The geometry shown in Fig. 1 is assumed, with $10 \mathrm{kV}$ applied to the conical repeller electrode and with a $26 \mathrm{~cm}$ separation between the repeller electrode and the detection plane. The ejection of the fragments is assumed to be isotropic. The diameter of the capillary tube that is integrated in the repeller electrode is $50 \mu \mathrm{m}$. The fragment source volume is assumed to be the intersection of an effusive flow with a Gaussian radial density distribution a 1 sr opening angle and a Gaussian laser beam that is focused to a spot size (FWHM) of $200 \mu \mathrm{m}$. The distance from the repeller electrode to the laser axis is assumed to be $1 \mathrm{~mm}$.

the distance from this flat part to the laser focus, since these two parameters determine both the fields experienced by the detected particles and the size of the interaction volume (taking into account that the sample effuses from the capillary).

Figures 4 and 5 show results that we have obtained in our simulations when assuming a $1 \mathrm{~mm}$ distance between the repeller and the laser focus and a $1 \mathrm{~mm}$ width of the flat part of the repeller. Similar to Fig. 2, Fig. 4(a) shows a simulated image, while Figs. 4(b) and 4(c) show a slice through the 3D velocity distribution and the kinetic energy distribution, respectively. As shown in Fig. 5, the new design shows a performance that compares quite satisfactorily with the performance of the Eppink and Parker $^{3}$ design. Clearly the resolution is somewhat degraded since the best performance $\Delta E / E=2.3 \%$ is now reached for fragments with a kinetic energy of $100 \mathrm{eV}$, compared to the best performance $\Delta E / E$ $=0.7 \%$ that was calculated in the open geometry, but otherwise the performance is quite comparable. A resolution of a few percent is acceptable for many of the applications where velocity map imaging would be considered.

In Fig. 6, the dependence of the energy resolution of the spectrometer is explored as a function of the distance from the repeller to the interaction region (a) and as a function of the width of the flat region of the repeller (b). In the former case the width of the flat region is held a $1 \mathrm{~mm}$, while in the latter case the distance from the repeller to the interaction region is held at $1 \mathrm{~mm}$. The kinetic energy resolution shows a clear dependence on the distance from the repeller to the interaction region. Below $1 \mathrm{~mm}$ the resolution significantly degrades since close to the repeller the new electrode shape introduces changes in the equipotential lines compared to the Eppink and Parker ${ }^{3}$ design. This means that experimentally 

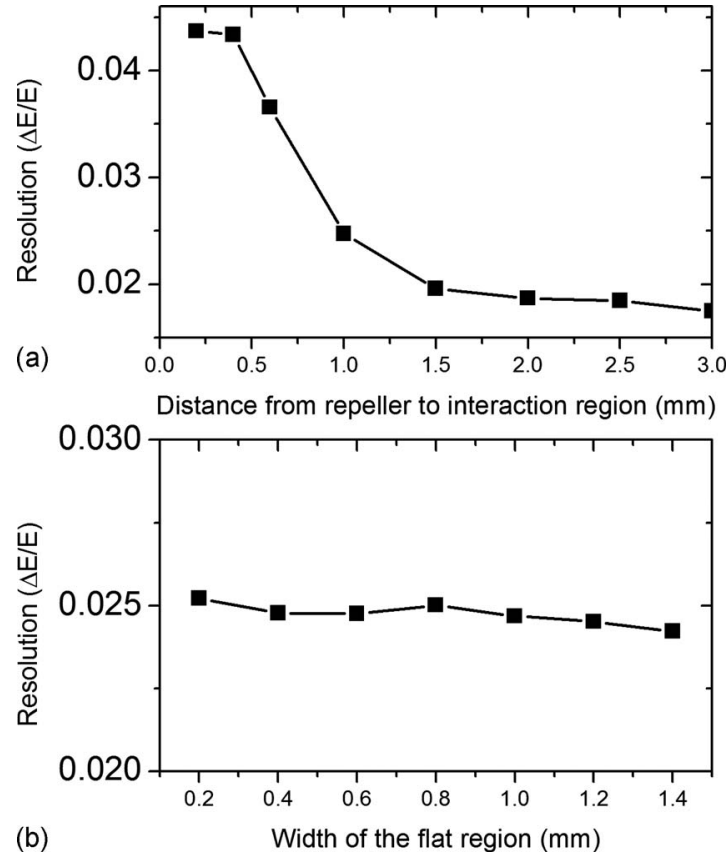

FIG. 6. Dependence of the calculated kinetic energy resolution of the velocity map imaging spectrometer as a function of (a) the distance from the repeller to the interaction region and (b) the width of the flat region of the repeller. In the former case the width of the flat region is held a $1 \mathrm{~mm}$, while in the latter case the distance from the repeller to the interaction region is held at $1 \mathrm{~mm}$. The kinetic energy resolution shows a clear dependence on the distance from the repeller to the interaction but does not show any dependence on the width of the flat region

there is a compromise between the energy resolution and the signal yield. The kinetic energy resolution shows only a slight dependence on the width of the flat region. Hence the parameters of our final design were chosen principally to allow the laser focus to be positioned close to the effusive source.

\section{EXPERIMENTAL TESTS}

In order to test the performance of the new velocity map imaging spectrometer, tests were performed where Xe atoms that were injected from the repeller assembly were ionized by the second harmonic of a Nd:YAG laser $(532 \mathrm{~nm})$. The photon energy $(2.33 \mathrm{eV})$ implies that at least six photons need to be absorbed in order to ionize the atom, which has an ionization potential of $12.13 \mathrm{eV}$. The measured images contain four contributions, corresponding to ionization that produces the $\mathrm{Xe}^{+}$ion in its ${ }^{2} P_{3 / 2}$ ground state or in the ${ }^{2} P_{1 / 2}$ spin-orbit excited state and using either six or seven photons of the Nd:YAG laser. The measured 2D image and a slice through the 3D velocity distribution that was obtained by means of the method in Ref. 15 are shown in Fig. 7. Also shown is a kinetic energy distribution that was obtained by integrating the $3 \mathrm{D}$ velocity distribution in a narrow cone $\left(5^{\circ}\right)$ along the polarization axis. The two peaks arising from seven-photon ionization are shown, which are both measured with a resolution of $\Delta E / E=1.8 \%$. Due to imperfections in the imaging that most likely can be attributed to the finite quality of the $\mu$-metal shield that surrounds the entire assembly, the observed images are not completely round, leading
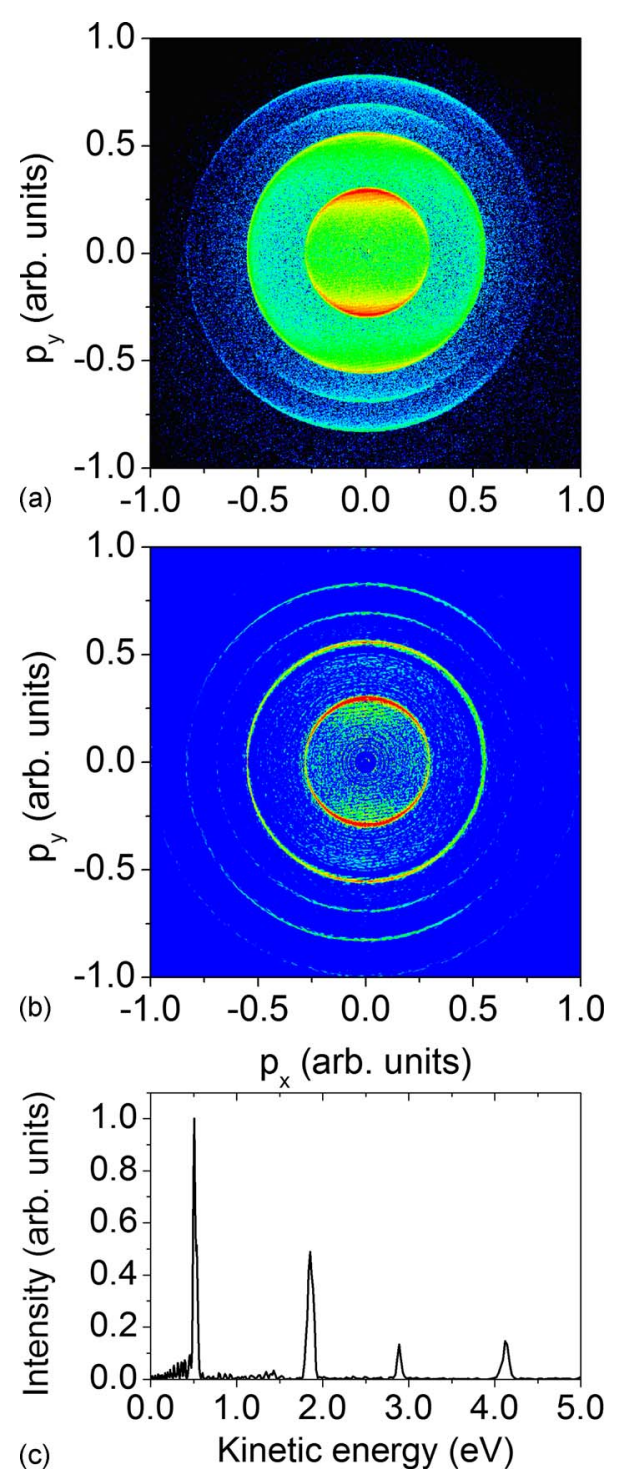

FIG. 7. (Color online) (a) Experimental photoelectron image obtained for six- and seven-photon ionization of Xe atoms using the second harmonic $(532 \mathrm{~nm})$ of a Nd:YAG laser. For each $n$-photon ionization process, two rings are observed that correspond to the formation of the $\mathrm{Xe}^{+}$ion in its ${ }^{2} P_{3 / 2}$ ground state or in the ${ }^{2} P_{1 / 2}$ spin-orbit excited state. (b) Slice through the $3 \mathrm{D}$ velocity distribution obtained by applying the iterative inversion procedure in Ref. 15. (c) Photoelectron kinetic energy distribution derived from integration of the $3 \mathrm{D}$ velocity distribution within a narrow cone $\left(5^{\circ}\right)$ along the polarization axis. Two contributions arising from seven-photon ionization are measured with a kinetic energy resolution of $\Delta E / E=1.8 \%$. When the photoelectron kinetic energy distribution is evaluated by full angular integration of the $3 \mathrm{D}$ velocity distribution, the resolution degrades to $5 \%$ due to imperfections in the roundness of the measured images, which are likely due to imperfections in the magnetic shielding of the spectrometer.

to a smearing of the kinetic energy distribution. If the 3D velocity distribution is integrated over the full angular range, the kinetic energy resolution degrades to $5 \%$.

A $1.8 \%$ kinetic energy resolution is very satisfactory and compares favorably to the performance that we had anticipated on the basis of our calculations. This is likely to be due to the fact that in multiphoton ionization, the ionization volume is not only limited by the presence of the sample (as was assumed in the calculation) but is furthermore limited by the focal volume of the laser. Under the conditions used in the experiment, the $532 \mathrm{~nm}$ was focused with $f / D=50$, lead- 
ing to a laser waist of $\omega_{0}=9 \mu \mathrm{m}$ and a Rayleigh range of $z_{R}=0.45 \mathrm{~mm}$, i.e., a somewhat smaller laser focus than we have assumed in our simulations. The achieved resolution is clearly sufficient for many of the applications that the present detector was designed for, such as using the output of very weak attosecond light sources that produce $10^{6}$ photons/pulse or less.

\section{ACKNOWLEDGMENTS}

This work is part of the research program of the "Stichting voor Fundamenteel Onderzoek der Materie (FOM)," which is financially supported by the "Nederlandse organisatie voor Wetenschappelijk Onderzoek (NWO).” M.F.K. acknowledges support by the Max-Planck Society and the Emmy-Noether program of the German Science Foundation (DFG). P.J. acknowledges the Swedish Research Council for a research fellowship.

${ }^{1}$ D. W. Chandler and P. L. Houston, J. Chem. Phys. 87, 1445 (1987).

${ }^{2}$ B. J. Whitaker, Imaging in Molecular Dynamics-Technology and Applications (A User's Guide) (Cambridge University Press, Cambridge, 2003).

${ }^{3}$ A. T. J. B. Eppink and D. H. Parker, Rev. Sci. Instrum. 68, 3477 (1997).

${ }^{4}$ T. Remetter, P. Johnsson, J. Mauritsson, K. Varju, Y. Ni, F. Lepine, E. Gustafsson, M. Kling, J. Khan, R. Lopez-Martens, K. J. Schafer, M. J. J. Vrakking, and A. L'Huillier, Nat. Phys. 2, 323 (2006).
${ }^{5}$ P. Johnsson, W. Siu, A. Gijsbertsen, J. Verhoeven, A. S. Meijer, W. van der Zande, and M. J. J.Vrakking, J. Mod. Opt. 55, 2693 (2008).

${ }^{6}$ C. R. Gebhardt, T. P. Rakitzis, P. C. Samartzis, V. Ladopoulos, and T. N. Kitsopoulos, Rev. Sci. Instrum. 72, 3848 (2001).

${ }^{7}$ D. Townsend, M. P. Minitti, and A. G. Suits, Rev. Sci. Instrum. 74, 2530 (2003).

${ }^{8}$ G. Sansone, E. Benedetti, F. Calegari, C. Vozzi, L. Avaldi, R. Flammini, L. Poletto, P. Villoresi, C. Altucci, R. Velotta, S. Stagira, S. De Silvestri, and M. Nisoli, Science 314, 443 (2006).

${ }^{9}$ E. Goulielmakis, M. Schultze, M. Hofstetter, V. S. Yakovlev, J. Gagnon, M. Uiberacker, A. L. Aquila, E. M. Gullikson, D. T. Attwood, R. Kienberger, F. Krausz, and U. Kleineberg, Science 320, 1614 (2008).

${ }^{10}$ P. C. Samartzis, D. J. Smith, T. P. Rakitzis, and T. N. Kitsopoulos, Chem. Phys. Lett. 324, 337 (2000).

${ }^{11}$ R. L. Toomes and T. N. Kitsopoulos, Phys. Chem. Chem. Phys. 5, 2481 (2003).

${ }^{12}$ F. Rosca-Pruna, Ph.D. thesis, AMOLF, 2001.

${ }^{13}$ J. Mauritsson, T. Remetter, M. Swoboda, K. Kluender, A. L'Huillier, K. Schafer, O. Ghafur, F. Kelkensberg, W. K. Siu, P. Johnsson, M. J. J. Vrakking, I. Znakovskaya, T. Uphues, S. Zherebtsov, M. F. Kling, F. Lepine, E. Benedetti, F. Ferrari, G. Sansone, and M. Nisoli (unpublished).

${ }^{14}$ G. Sansone, F. Kelkensberg, M. Kling, W. Siu, O. Ghafur, P. Johnsson, M. Swoboda, E. Benedetti, F. Ferrari, F. Lepine, S. Zherebtsov, I. Znakovskaya, A. L'Huillier, M. Ivanov, M. Nisoli, and M. J. J. Vrakking (unpublished).

${ }^{15}$ M. J. J. Vrakking, Rev. Sci. Instrum. 72, 4084 (2001).

${ }^{16}$ M. L. Lipciuc, J. B. Buijs, and M. H. M. Janssen, Phys. Chem. Chem. Phys. 8, 219 (2006). 